\title{
The safety and efficacy of haemostasis with a catechol-conjugated, chitosan-based haemostatic dressing versus a chitosan-based haemostatic dressing after transfemoral approach for transcatheter arterial chemoembolization: a randomized controlled trial
}

\author{
Sang Mi Seol ${ }^{1, A, B, D, E, F *}$, Pyeong Hwa Kim ${ }^{1, B, C, D, E, F *}$, Ji Hoon Shin ${ }^{1, A, D, E, F}$, Seng Yong Chun ${ }^{1, B}$, Mi Young Lee ${ }^{1, B}$, \\ Kwang Mi Kim ${ }^{1, B}$, Hyun-Ki Yoon ${ }^{1, A, E}$ \\ Department of Radiology, University of Ulsan, College of Medicine, Asan Medical Centre, Seoul, South Korea \\ *These authors contributed equally to this work.
}

\section{Abstract}

\begin{abstract}
Purpose: To compare the haemostatic efficacy (i.e. efficacy to prevent access site complications) of the InnoSEAL haemostatic pad and Clo-Sur PLUS P.A.D. after femoral arterial puncture for transcatheter arterial chemoembolization (TACE).

Material and methods: This randomized controlled trial compared the safety and efficacy of an InnoSEAL haemostatic pad $(n=48)$ and a Clo-Sur PLUS P.A.D. $(n=52)$ for haemostasis of arterial puncture sites after TACE with femoral arterial access using a 5-Fr sheath. Primary endpoints were incidence of major (necessitating surgery) and moderate access site complications (ASC) (necessitating blood transfusion/thrombin injection). Secondary endpoints were incidence of minor ASC (no therapy required) and time to haemostasis.

Results: No major or moderate ASC was seen with either device. Minor ASC (6.3\% [3/48] vs. 19.2\% [10/52], $p=0.075)$ and ecchymosis (classified as minor ASC; $4.2 \%$ [2/48] vs. 17.3\% [9/52]; $p=0.053$, $p$-value cut-off after Bonferroni correction $=0.025$ ) were less frequently observed with the InnoSEAL haemostatic pad. The time to haemostasis did not differ significantly between the 2 devices ( $5.6 \pm 1.0$ vs. $5.3 \pm 0.7$ minutes; $p=0.118$ ). Multivariable logistic regression analysis showed a lower risk of ASC with the InnoSEAL pad (adjusted OR, 0.174; 95\% CI: 0.034-0.890; $p=0.036$ ).
\end{abstract}

Conclusions: No major ASC was seen with either pad, and no significant difference of minor ASC was observed between 2 pads.

Key words: chemoembolization, femoral artery, access site complications, haemostatic pad, randomized controlled trial.

\section{Introduction}

Transcatheter arterial chemoembolization (TACE) is a widespread therapeutic option for hepatocellular carcinoma, and femoral arterial puncture is the most frequent means of access to the vascular system for TACE.
Despite technical advances in catheter systems, which have enabled decreases in sheath sizes, access site complications (ASC) remain the most frequent adverse events after percutaneous transluminal procedures, comprising up to $11 \%$ of them [1]. Furthermore, ASCs are occasionally a significant cause of morbidity, leading to prolonged

Correspondence address:

Ji Hoon Shin, Department of Radiology, University of Ulsan, College of Medicine, Asan Medical Centre, 88 Olympic-ro 43-gil, Songpa-gu, Seoul, South Korea, e-mail: jhshin@amc.seoul.kr

Authors' contribution:

A Study design · B Data collection · C Statistical analysis · D Data interpretation · E Manuscript preparation · F Literature search · G Funds collection 
hospital stay and an increase in the mortality rate [2]. Therefore, appropriate management of ASCs after TACE remains an important issue.

Traditionally, among the available methods for haemostasis, manual compression has been considered as the standard; however, it is time-consuming, personally intensive, requires prolonged bed rest, causes inconvenience to both patients and performers, and can be an obstacle preventing the efficient use of angiography suite resources. Compared with manual compression, mechanical closure devices have advantages in terms of a short turnaround time and expectations of less frequent ASCs, but they are expensive, and only a marginally lower complication rate has been demonstrated [3]. In recent decades, topically applied haemostatic pads have been widely used to achieve access site haemostasis because they are particularly easy to handle without requiring the placement of intra- or extravascular foreign materials, thereby enabling immediate arterial re-puncture and avoiding the risk of devicerelated complications $[2,4,5]$. Among the commercially available haemostatic pads, a chitosan-based haemostatic dressing (Clo-Sur PLUS P.A.D.) has been reported to offer a shorter time to haemostasis without any difference in bleeding complication rates compared with manual compression without a chitosan-based pad [6,7]. Recently, a catechol-conjugated chitosan-based haemostatic dressing (InnoSEAL haemostatic pad) has been widely used because it provides an additional antimicrobial barrier effect.

Therefore, this RCT was performed to compare the safety and efficacy of the InnoSEAL haemostatic pad and the Clo-Sur PLUS P.A.D. in achieving haemostasis after femoral arterial puncture for transcatheter arterial chemoembolization (TACE).

\section{Material and methods}

This single-centre open-label RCT was approved by our institutional review board, and written informed consent was obtained from each participant. This study was conducted in accordance with the CONSORT statement [8].

\section{Patient eligibility, enrolment, and randomization}

From June to August 2017, patients aged between 20 and 80 years, who underwent TACE with femoral arterial access using a 5-Fr sheath were eligible for participation in the study. We only included the first patient of the day to ensure the same standard of care by the trained nurses during the daytime. Patients who were allergic to chitosan, which was determined by asking the patient if he or she was allergic to crustaceans (which contain chitosan), were excluded. No patients received a platelet transfusion just before the TACE, and all the participants were prohibited from taking any medications that could provoke bleeding diathesis (i.e. aspirin, antiplatelet agent, or anticoagulants) for at least 7 days before TACE.
Randomization in a 1:1 ratio was performed at the study design stage by a researcher who did not participate in either recruitment of the study participants or data evaluation. Computer-generated random digits ( 1 indicating the InnoSEAL haemostatic pad and 0 the Clo-Sur PLUS P.A.D.) and sealed envelopes were used to assign study participants to each group. Our target sample size was 100 patients (50 patients per group), and assuming a $10 \%$ dropout rate, a total of 110 envelopes (100 and additional 10 envelopes as spares) were prepared in advance. The envelopes were opened in order just after TACE.

\section{Haemostasis procedure}

During the TACE, the right femoral artery was punctured based on the point of maximum pulsation by interventional radiologists with at least 10 years of experience in TACE without ultrasound guidance. In all patients, the pulsation at the right femoral artery and right dorsalis pedis artery was checked before the puncture. If pulsation was poor, the contralateral side was checked, and a puncture was tried if it was patent. After completion of TACE, the inserted 5-Fr sheath was removed. The puncture site was manually compressed by applying the randomly assigned haemostatic pads (InnoSEAL haemostatic pad; Innotherapy, Seoul, Korea] vs. Clo-Sur PLUS PAD [Medtronic Vascular, Santa Rosa, CA, USA]) in accordance with the manufacturer's instructions for use (Figure 1). Mechanical closure devices (e.g. disc, plug, metal clip, or suture-based devices) were not used in this study, these being reserved for procedures using a sheath of 7-Fr or larger, according to our institution's policy. Manual compression was performed on all study participants by clinically experienced registered nurses. In detail, a total of 10 nurses specialized in access site management and with at least 3 years of experience participated in this study. The nurses were also pre-trained for standardization, including maintaining a pressure such that the dorsalis pedis artery could be palpated. According to the instructions given in the product manual, manual compression was maintained for 5 minutes, after which the puncture site was assessed for haemostasis. If there was no bleeding from the puncture site, the patient was instructed to perform a cough to confirm haemostasis. If there was bleeding from the puncture site, compression was applied for an additional minute and the puncture site haemostasis was then reassessed.

After achievement of complete haemostasis, patients were transferred to the ward with a bandage and a sandbag applied to the device. The groin site was reassessed 3 hours after the transfer, and patients without recurrent bleeding from the puncture site were encouraged to ambulate. If recurrent bleeding was observed, ambulation was planned to be delayed for 2 hours and the patient was reassessed for recurrent bleeding. The haemostatic device was then removed in the ward after 24 hours of haemostasis, following the instructions given in the product manuals. One week 
after the procedure, the patient was re-evaluated by the aforementioned nurses specialized in access site management to identify the occurrence of post-procedural complications, e.g. haematoma, ecchymosis, and pain.

\section{Data collection, outcomes, and statistical analysis}

The following data were collected: patient age, year, underlying medical conditions (e.g. hypertension, diabetes mellitus, tobacco smoking, use of aspirin, antiplatelet agent, and/ or anticoagulants), the number of previous TACE procedures, platelet count, prothrombin time (PT) and activated partial thromboplastin time (aPTT) just before the procedure, presence of coagulopathy (defined as platelet count $<50,000 / \mu$ or international normalized ratio [INR] $\geq 1.5$ ), blood pressure just after the procedure, and the number of puncture attempts during the procedure.

The primary study endpoints were the incidence of major ASC (necessitating surgery) and moderate ASC (necessitating blood transfusion and/or thrombin injection) [2]. Secondary study endpoints were the incidence of minor ASCs (no additional therapy used, including self-limiting recurrent bleeding, ecchymosis, or haematoma) and the time to haemostasis (time interval from vascular sheath removal to cessation of bleeding from the femoral arterial puncture site). Incidence rates of major, moderate, and minor ASCs were compared between the 2 groups using Fisher's exact test or the chi-square test as appropriate. Additionally, the risk of ASCs between the 2 groups was compared using multivariable logistic regression analysis. The baseline characteristics (Table 1) were first screened using univariable logistic regression analyses, and variables with $p$-values $<0.1$ in the univariable analyses, and age, sex, body mass index, presence of hypertension, and renal function (representative value as estimated by glomerular filtration rate [eGFR]) [9-12], which are well accepted as risk factors for vascular access site bleeding, as well as platelet count and INR, which are indicative markers for coagulopathy, were included as covariates in the multivariable analysis.

Basically, a $p$-value of $<0.05$ was considered to indicate a statistically significant difference, and a Bonferroni correction for the significance level was applied when multiple factors were considered as minor ASC, such as the incidence of recurrent bleeding, access site haematoma, and ecchymosis. Statistical analyses were conducted using SPSS version 23 (IBM, Chicago, IL, USA), and post hoc power calculations were conducted using PASS 2019 (NCSS, Kaysville, Utah).

\section{Results}

\section{Demographics and clinical characteristics}

A patient selection flowchart is presented in Figure 2. A total of 138 patients underwent TACE as the first sched-

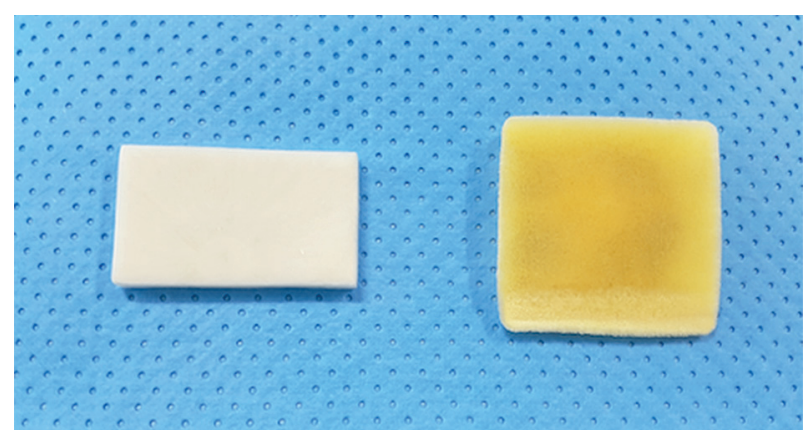

Figure 1. An InnoSEAL haemostatic pad (left; $5 \times 3 \mathrm{~cm}$ ) and Clo-Sur PLUS P.A.D. (right; $4 \times 4 \mathrm{~cm}$ )

ule of the day during the study period. Of these, $35 \mathrm{pa}-$ tients were excluded for the following reasons: (a) did not agree to participate $(n=25)$; (b) aged over 80 years $(n=5)$; and (c) body weight over $90 \mathrm{~kg}(n=5)$. Consequently, a total of 100 patients (48 with an InnoSEAL haemostatic pad [mean age \pm SD, $57.8 \pm 8.5$ years; 44 men and 4 women] and 52 with a Clo-Sur PLUS P.A.D. (mean age \pm SD, $62.6 \pm 7.4$ years; 46 men and 6 women) were included in the analysis. The right femoral artery was punctured in all included patients because the pulsation of the dorsalis pedis artery was patent. There were 2 patients who used aspirin for the secondary prevention of coronary artery disease in the InnSEAL haemostatic pad group and 2 in the Clo-Sur P.A.D. group. One patient in the InnSEAL haemostatic pad group used clopidogrel alone for the secondary prevention of cerebral infarction, and 2 patients in the Clo-Sur PLUS P.A.D. group used aspirin and clopidogrel for atrial fibrillation. There were 3 patients with coagulopathy in the InnoSEAL haemostatic pad group, but no patients in the Clo-Sur PLUS P.A.D. group had coagulopathy $(6.2 \%$ [3/48] vs. $0 \%$ [0/52]; all 3 patients showed a platelet count $<50,000$ per $\mu$ l but an INR $<1.5)$. When comparing the 2 groups, patient age $(p=0.003)$ and the proportion of current smokers $(p=0.046)$ were statistically different. The other variables did not show statistical differences between the 2 groups (Table 1).

\section{Outcomes and risk of ASC between the two groups}

There was no major or moderate ASC in either group. Overall, ASC was less frequently observed in the InnoSEAL haemostatic pad group than in the Clo-Sur PLUS P.A.D. group, showing a tendency toward statistical significance $(6.3 \%$ [3/48] vs. $19.2 \%$ [10/52], $p=0.075)$. In detail, an access site haematoma developed in 1 patient in each group (2.1\% [1/48] vs. $1.9 \%[1 / 52] ; p=1.000)$, and these were self-limiting. Ecchymosis was less frequently observed in the InnoSEAL group than in the Clo-Sur PLUS P.A.D. group (4.2\% [2/48] vs. $17.3 \%$ [9/52]; $p=$ 0.053 , with a cut-off $p$-value of 0.025 after Bonferroni correction), although the difference did not reach statistical significance. The time to haemostasis did not differ significantly between the InnoSEAL and Clo-Sur PLUS 
Table 1. Baseline characteristics of the included patients

\begin{tabular}{|c|c|c|c|}
\hline Characteristic & $\begin{array}{l}\text { InnoSEAL haemostatic pad } \\
\qquad(n=48)\end{array}$ & $\begin{array}{l}\text { Clo-Sur PLUS P.A.D. } \\
\qquad(n=52)\end{array}$ & $p$-value \\
\hline Age, mean $\pm S D$ & $57.8 \pm 8.5$ & $62.6 \pm 7.4$ & 0.003 \\
\hline \multicolumn{4}{|l|}{ Sex, $n(\%)$} \\
\hline Male & $44(91.7)$ & $46(88.5)$ & \multirow[t]{2}{*}{0.287} \\
\hline Female & $4(8.3)$ & $6(11.5)$ & \\
\hline Weight $(\mathrm{kg})$, mean \pm SD & $68.6 \pm 9.1$ & $66.5 \pm 10.4$ & 0.303 \\
\hline BMI $\left(\mathrm{kg} / \mathrm{m}^{2}\right)$, mean \pm SD & $24.5 \pm 3.0$ & $24.4 \pm 3.4$ & 0.895 \\
\hline Hypertension, $n(\%)$ & $21(43.8)$ & $19(36.5)$ & 0.541 \\
\hline Diabetes mellitus, $n(\%)$ & $14(29.2)$ & $13(25.0)$ & 0.220 \\
\hline \multicolumn{4}{|l|}{ Drug history, $n(\%)$} \\
\hline Aspirin & $2(4.2)$ & $4(7.7)$ & 0.679 \\
\hline Clopidogrel & $1(2.1)$ & $2(3.8)$ & 1.000 \\
\hline \multicolumn{4}{|l|}{ Tobacco use, $n(\%)$} \\
\hline None & $14(29.2)$ & $21(40.4)$ & 0.334 \\
\hline Ex-smoker & $21(43.8)$ & $25(48.1)$ & 0.664 \\
\hline Current smoker & $13(27.1)$ & $6(11.5)$ & 0.046 \\
\hline Platelet count $\left(10^{3} / \mu \mathrm{l}\right)$, mean \pm SD & $119.2 \pm 46.3$ & $139.8 \pm 65.5$ & 0.075 \\
\hline PT $(\mathrm{s})$, mean \pm SD & $81.5 \pm 12.1$ & $84.6 \pm 11.4$ & 0.191 \\
\hline INR, mean \pm SD & $1.1 \pm 0.1$ & $1.1 \pm 0.1$ & 0.154 \\
\hline aPTT (s), mean \pm SD & $27.1 \pm 4.9$ & $27.2 \pm 4.0$ & 0.948 \\
\hline eGFR $\left(\mathrm{ml} / \mathrm{min} / 1.73 \mathrm{~m}^{2}\right)$, mean \pm SD & $92.7 \pm 17.4$ & $87.6 \pm 14.1$ & 0.111 \\
\hline Coagulopathy ${ }^{\dagger}, n(\%)$ & $3(6.2)$ & $0(0)$ & 0.107 \\
\hline No. of previous TACEs, median (range) & $3.7(0-25)$ & $4.0(0-11)$ & 0.690 \\
\hline No. of punctures during the TACE, median (range) & $1.0(1-3)$ & $1.1(1-3)$ & 0.279 \\
\hline sBP just after the TACE (mmHg), mean \pm SD & $121.1 \pm 15.5$ & $125 \pm 17.8$ & 0.225 \\
\hline dBP just after the TACE (mmHg), mean \pm SD & $77.2 \pm 8.9$ & $77.5 \pm 9.7$ & 0.877 \\
\hline
\end{tabular}

${ }^{\dagger}$ Defined as platelet count $<50,000$ per $\mu$ l or INR $\geq 1.5$.

BMI - body mass index, DM - diabetes mellitus, PT - prothrombin time, INR - international normalized ratio, aPTT - activated partial thromboplastin time, eGFR - estimated glomerular filtration rate, $\mathrm{SBP}$ - systolic blood pressure, dBP - diastolic blood pressure

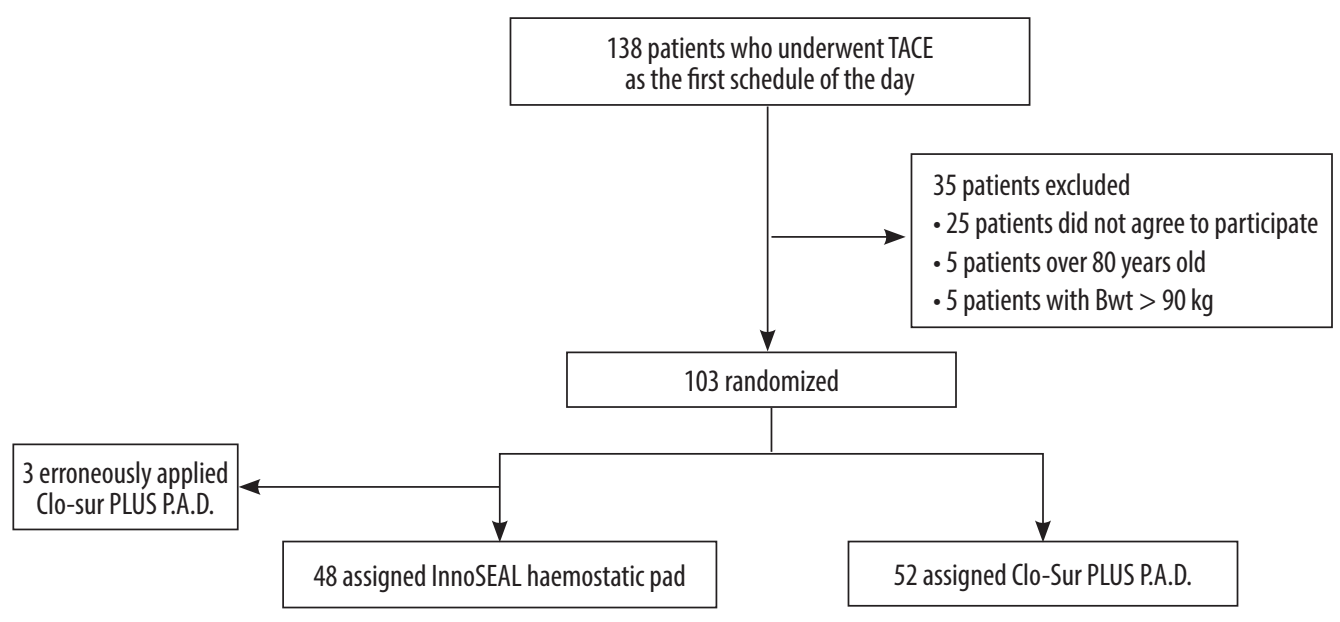

Figure 2. CONSORT diagram of the trial 
P.A.D. groups ( $5.6 \pm 1.0$ vs. $5.3 \pm 0.7$ minutes; $p=0.118$ ), and there was no clinically relevant recurrent bleeding in either group.

In the logistic regression analysis, the unadjusted odds ratio between the InnoSEAL and Clo-Sur PLUS P.A.D. groups for the risk of ASCs was 0.280 (95\% CI: 0.072-1.088; $p=0.066)$, and the adjusted odds ratio was $0.174(95 \% \mathrm{CI}$ : $0.034-0.890 ; p=0.036$ ). In addition, the use of clopidogrel significantly increased the incidence of ASCs (adjusted OR, 28.848; 95\% CI: 1.798-462.813; $p=0.018$; Table 2).

Table 2. Multivariable logistic regression analysis of the risk of femoral artery access site complications (i.e., hematoma, ecchymosis) in the InnoSEAL group compared with the Clo-Sur PLUS P.A.D. group

\begin{tabular}{|c|c|c|c|c|c|c|}
\hline \multirow[t]{2}{*}{ Parameter } & \multicolumn{3}{|c|}{ Univariable analysis } & \multicolumn{3}{|c|}{ Multivariable analysis } \\
\hline & Unadjusted OR & $95 \% \mathrm{Cl}$ & $p$-value & Adjusted OR & $95 \% \mathrm{Cl}$ & $p$-value \\
\hline \multicolumn{7}{|l|}{ Main factor } \\
\hline \multicolumn{7}{|l|}{ Types of hemostatic pad } \\
\hline Clo-Sur PLUS & Reference category & & & Reference category & & \\
\hline InnoSEAL & 0.280 & $0.072-1.088$ & 0.066 & 0.174 & $0.034-0.890$ & 0.036 \\
\hline \multicolumn{7}{|l|}{ Covariates } \\
\hline Age & 1.025 & $0.955-1.101$ & 0.491 & 1.000 & $0.912-1.096$ & 0.998 \\
\hline \multicolumn{7}{|l|}{ Sex } \\
\hline Male & Reference category & & & Reference category & & \\
\hline Female & 1.795 & $0.337-9.566$ & 0.493 & 1.418 & $0.167-12.051$ & 0.749 \\
\hline BMI $\left(\mathrm{kg} / \mathrm{m}^{2}\right)$ & 1.047 & $0.871-1.260$ & 0.624 & 1.032 & $0.823-1.295$ & 0.784 \\
\hline \multicolumn{7}{|l|}{ Hypertension } \\
\hline № & Reference category & & & Reference category & & \\
\hline Yes & 1.336 & $0.414-4.315$ & 0.628 & 1.862 & $0.417-8.323$ & 0.416 \\
\hline Diabetes mellitus & 1.237 & $0.347-4.406$ & 0.743 & & & \\
\hline \multicolumn{7}{|l|}{ Use of aspirin } \\
\hline No & Reference category & & & & & \\
\hline Yes & 3.773 & $0.617-23.056$ & 0.151 & & & \\
\hline \multicolumn{7}{|l|}{ Use of clopidogrel } \\
\hline No & Reference category & & & Reference category & & \\
\hline Yes & 15.636 & $1.308-186.932$ & 0.030 & 28.848 & $1.798-462.813$ & 0.018 \\
\hline \multicolumn{7}{|l|}{ Current smoker } \\
\hline No & Reference category & & & & & \\
\hline Yes & 0.319 & $0.039-2.621$ & 0.288 & & & \\
\hline Platelet count $\left(10^{3} / \mu \mathrm{l}\right)$ & 0.995 & $0.984-1.007$ & 0.439 & 0.991 & 0.976-1.007 & 0.275 \\
\hline PT $(s)$ & 0.980 & $0.932-1.031$ & 0.433 & & & \\
\hline INR & 5.647 & $0.014-2349.732$ & 0.574 & 21.590 & $0.013-34938.509$ & 0.415 \\
\hline $\mathrm{eGFR}\left(\mathrm{ml} / \mathrm{min} / 1.73 \mathrm{~m}^{2}\right)$ & 1.001 & $0.965-1.039$ & 0.950 & 1.016 & $0.966-1.068$ & 0.539 \\
\hline No. of previous TACEs & 1.061 & $0.926-1.217$ & 0.392 & & & \\
\hline $\begin{array}{l}\text { No. of punctures during } \\
\text { the TACE }\end{array}$ & 1.753 & $0.457-6.720$ & 0.413 & & & \\
\hline
\end{tabular}

aPTT was not included in the analysis due to missing values in eight patients.

The variables with $p$-values $<0.1$ in the univariable analyses and age, sex, body mass index, hypertension, use of aspirin and/or antiplatelet agent, which are well agreed upon as risk factors for vascular access site bleeding, and platelet count and INR, which are indicative markers for coagulopathy, were included as covariates in the multivariable analysis.

BMI - body mass index, DM - diabetes mellitus, PT - prothrombin time, INR - international normalized ratio, aPTT - activated partial thromboplastin time, eGFR - estimated glomerular filtration rate, $\mathrm{SBP}$ - systolic blood pressure, $\mathrm{dBP}$ - diastolic blood pressure 


\section{Discussion}

This single-centre RCT showed that (a) no major ASC was seen in either group; (b) the minor ASC rate was insignificantly lower in the InnoSEAL group than in the Clo-Sur PLUS P.A.D group; (c) the time to haemostasis did not differ between the 2 groups; and (d) the InnoSEAL group showed a lower risk of ASC in the multivariable logistic regression analysis. Future trials with sample size and power analysis calculations to ensure sufficient patient numbers are required to confirm this preliminary data.

As percutaneous interventional procedures become increasingly used, management of access site bleeding emerges as an important clinical issue. In particular, because many patients receiving TACE have a bleeding tendency due to hepatic dysfunction, access site management in these patients requires special attention. A variety of arterial closure devices have been commercially introduced, and these can be broadly categorized as mechanical closure devices, compression assist devices, and external topical haemostasis devices [13,14]. Mechanical closure devices are preferentially adopted by many operators because they can offer short turnaround time with the expectation that ASCs will be less frequent than with manual compression, and a meta-analysis including 34 studies showed only marginally fewer complications compared with manual compression [3]. Topically applied haemostatic pads, including the Clo-Sur P.A.D. and Clo-Sur PLUS P.A.D., have been commonly used because of their convenience and respectable haemostatic effect $[15,16]$. Chitosanbased pads, including the Clo-Sur P.A.D., promote clot formation by attracting negatively charged red blood cells and platelets to the positively charged chitosan molecules, and offer a shorter time to haemostasis without any difference in bleeding complication rates compared with manual compression without chitosan-based pads [6,7]. Indeed, 2 RCTs showed shorter ambulation times with similar bleeding complication rates after percutaneous procedures using a Clo-Sur P.A.D. in comparison with manual compression. The InnoSEAL haemostatic pad differs from the Clo-Sur P.A.D or Clo-Sur PLUS P.A.D. in that it uses catechol-conjugated chitosan. Theoretically, the catechol groups induce stable tissue adhesion, thus providing an adjunctive haemostatic effect [17]. In addition, the InnoSEAL pad contains a highly porous sponge, leading to rapid blood absorption and effective haemostasis. In this context, it is necessary to evaluate the safety and efficacy of this new chitosan-based haemostatic pad.
The logistic regression analysis showed that use of the InnoSEAL haemostatic pad resulted in a significantly decreased risk of ASCs compared with use of the Clo-Sur PLUS P.A.D. (adjusted odds ratio, 0.174; 95\% CI: 0.0340.890 ), which seemed to show discrepancy with our main results. However, the dependent variable in this logistic regression analysis was overall ASC (including ecchymosis), not major or moderate ASC. Further RCTs may clarify this issue. In addition, a comparison with mechanical closure devices also seems clinically worthwhile.

This study has several limitations of note. First, a blinded study design was impossible because of the distinct appearance of the different haemostatic pads. Second, because this study was conducted as a pilot study without a sample size calculation in the study design, there was a risk of the statistical analyses being underpowered. Post hoc power calculation revealed a power of 0.51 to detect an effect size $\geq 0.129$ (difference of minor ASC rates between 2 groups) with a sample size of 50 per group and a significance level of 0.05 , and conversely a sample size of 100 participants per group is required to detect an effect size $\geq 0.129$ with a power of 0.80 and a significance level of 0.05 . Thus, a further RCT including at least 100 participants per group is required for definitive results. However, according to the statistical rule of thumb of 5 or 10 patients per variable [18], the inclusion of 9 independent variables in the multivariable regression analysis to evaluate the risk of ASCs was tolerable. Finally, because only a minor proportion of the patients had coagulopathy (3\%) and all patients were prohibited from taking any medications that could provoke bleeding diathesis before the procedure, the generalization of our results to the clinical setting of coagulopathy is limited. Further studies to evaluate the haemostatic efficacy of InnoSEAL haemostatic pads in the general clinical setting of coagulopathy are mandatory.

\section{Conclusions}

No major or moderate ASC occurred after TACE in either the InnoSEAL or Clo-sur PLUS P.A.D groups. No significant difference of minor ASC was observed between the 2 pads.

\section{Conflict of interest}

The authors report no conflict of interest.

References

1. Noori VJ, Eldrup-Jorgensen J. A systematic review of vascular closure devices for femoral artery puncture sites. J Vasc Surg 2018; 68: 887-899.
2. Ortiz D, Jahangir A, Singh M, et al. Access site complications after peripheral vascular interventions: incidence, predictors, and outcomes. Circ Cardiovasc Interv 2014; 7: 821-828. 
3. Das R, Ahmed K, Athanasiou T, et al. Arterial closure devices versus manual compression for femoral haemostasis in interventional radiological procedures: a systematic review and meta-analysis. Cardiovasc Intervent Radiol 2011; 34: 723-738.

4. Mlekusch W, Minar E, Dick P, et al. Access site management after peripheral percutaneous transluminal procedures: neptune pad compared with conventional manual compression. Radiology 2008; 249 : 1058-1063.

5. Kordestani SS, Noohi F, Azarnik H, et al. A randomized controlled trial on the hemostasis of femoral artery using topical hemostatic agent. Clin Appl Thromb Hemost 2012; 18: 501-505.

6. Mlekusch W, Dick P, Haumer M, et al. Arterial puncture site management after percutaneous transluminal procedures using a hemostatic wound dressing (Clo-Sur P.A.D.) versus conventional manual compression: a randomized controlled trial. J Endovasc Ther 2006; 13: $23-31$

7. Nguyen N, Hasan S, Caufield L, et al. Randomized controlled trial of topical hemostasis pad use for achieving vascular hemostasis following percutaneous coronary intervention. Catheter Cardiovasc Interv 2007; 69: 801-807.

8. Schulz KF, Altman DG, Moher D, et al. CONSORT 2010 statement: updated guidelines for reporting parallel group randomised trials. BMJ 2010; 340: c332.

9. Doyle BJ, Ting HH, Bell MR, et al. Major femoral bleeding complications after percutaneous coronary intervention: incidence, predictors, and impact on long-term survival among 17,901 patients treated at the Mayo Clinic from 1994 to 2005. JACC Cardiovasc Interv 2008; 1: 202-209.
10. Nikolsky E, Mehran R, Dangas G, et al. Development and validation of a prognostic risk score for major bleeding in patients undergoing percutaneous coronary intervention via the femoral approach. Eur Heart J 2007; 28: 1936-1945.

11. Tavris DR, Wang Y, Jacobs S, et al. Bleeding and vascular complications at the femoral access site following percutaneous coronary intervention (PCI): an evaluation of hemostasis strategies. J Invasive Cardiol 2012; 24: 328-334.

12. Lee MS, Applegate B, Rao SV, et al. Minimizing femoral artery access complications during percutaneous coronary intervention: a comprehensive review. Catheter Cardiovasc Interv 2014; 84: 62-69.

13. Sheth RA, Walker TG, Saad WE, et al. Quality improvement guidelines for vascular access and closure device use. J Vasc Interv Radiol 2014; 25: 73-84.

14. Schwartz BG, Burstein S, Economides C, et al. Review of vascular closure devices. J Invasive Cardiol 2010; 22: 599-607.

15. Balzer JO, Schwarz W, Thalhammer A, et al. Postinterventional percutaneous closure of femoral artery access sites using the Clo-Sur PAD device: initial findings. Eur Radiol 2007; 17: 693-700.

16. Choi EY, Ko YG, Kim JB, et al. Hemostatic efficacy of hydrophilic wound dressing after transradial catheterization. J Invasive Cardiol 2005; 17: 459-462.

17. Kim K, Ryu JH, Lee DY, et al. Bio-inspired catechol conjugation converts water-insoluble chitosan into a highly water-soluble, adhesive chitosan derivative for hydrogels and LbL assembly. Biomater Sci 2013; 1: 783-790.

18. Vittinghoff E, McCulloch CE. Relaxing the rule of ten events per variable in logistic and Cox regression. Am J Epidemiol 2007; 165: 710-718. 